\title{
LA MUJER MARROQUÍ Y LOS OBJETIVOS DE DESARROLLO DEL MILENIO
}

\section{THE MOROCCAN WOMAN AND THE MILLENNIUM DEVELOPMENT GOALS}

\author{
Mohamed Laghdas \\ Universidad de Granada, Granada. España/Spain \\ mohamed.laghdas@gmail.com
}

Recibido/Received: 19/04/2017

Modificado/Modified: 26/07/2017

Aceptado/Accepted: 05/10/2017

\section{RESUMEN}

En las últimas décadas, la situación de la mujer marroquí ha conocido un cambio a nivel social, económico y político, lo que ha propiciado el camino hacia la consecución de un desarrollo sostenible. En este sentido, Marruecos se comprometió a lograr los ocho Objetivos de Desarrollo del Milenio (ODM) entre los que se encuentran dos directamente relacionados con la mujer, que son el tercero y el quinto. El objetivo de este artículo es analizar los esfuerzos y los avances realizados mediante datos cualitativos y cuantitativos y centrándonos en el ámbito de la promoción de la igualdad entre géneros y la autonomía de la mujer, así como la mejora de la salud materna.

\section{PALABRAS CLAVE}

Marruecos; autonomía de la mujer; adopción de decisiones; igualdad; salud materna.

\section{SUMARIO}

1. Introducción. 2. Promoción de la igualdad entre géneros y la autonomía de la mujer. 2.1. La igualdad en la educación. 2.2. La igualdad en el mundo laboral. 2.3. La participación en los procesos de adopción de decisiones. 2.4. Modificaciones en el arsenal jurídico. 3. La mejora de la salud materna. 3.1. El serio problema de la mortalidad materna en países en vía de desarrollo. 3.2. La evolución gradual en la mejora de la salud materna en Marruecos. 4. Conclusión. Bibliografía.

\begin{abstract}
In the last decades, the situation of the Moroccan woman has experienced a change at social, economic, and politic level, which has led to a path to attain sustainable development. Regarding this, Morocco committed to achieving the eight Millennium Development Goals (MDGs) including two directly related to women, which are the third and fifth. The objective of this article is to analyze the efforts and the advances made in the field of the promotion of gender equality and the autonomy of women by means of qualitative and quantitative data, as well as the improvement of maternal health.
\end{abstract}

\section{KEYWORDS}

Morocco; women's autonomy; decision-making; equality; maternal health. 


\section{CONTENTS}

1. Introduction. 2. Promotion of gender equality and women's empowerment. 2.1. Equality in education. 2.2. Equality in the world of work. 2.3. Participation in decision-making processes. 2.4. Modifications in the legal arsenal. 3. Improving maternal health. 3.1. The serious problem of maternal mortality in developing countries. 3.2. The gradual evolution in the improvement of maternal health in Morocco. 4. Conclusion. References.

\section{INTRODUCCIÓN}

Después de la independencia, Marruecos conoció una recesión económica por la fuga de capitales, lo que llevó a la disminución de la productividad y a la rentabilidad de los sectores agrícola e industrial. A nivel social, uno de los fenómenos más destacados después de la independencia era la pobreza que vivía la mayoría de la población, con desigualdades sociales dentro de las ciudades y también entre ciudades y pueblos, a lo que sumamos que el país comenzó a sufrir una presión demográfica. En tales circunstancias, a pesar de adoptar varios planes de desarrollo económico y social, éstos no alcanzaron gran éxito, sino que con el aumento de la deuda y la aplicación del Programa de Ajuste Estructural en los años ochenta y noventa, tuvieron repercusiones negativas a nivel social, como el aumento del desempleo o la pobreza.

En el año 2000, y exactamente en septiembre, se firmó el acuerdo más amplio de la Comunidad Internacional con el fin de la lucha contra la pobreza, el hambre, la enfermedad, el analfabetismo, la degradación del medio ambiente y la discriminación contra la mujer, que a nuestro entender se consideran necesidades y derechos humanos de máxima prioridad. Así, se establecieron de manera común un conjunto de propósitos llamados Objetivos de Desarrollo del Milenio (ODM, en adelante), que todos los miembros de la Asamblea General las Naciones Unidas convinieron en tratar de alcanzar para 2015. Ésta fue la primera vez que la Organización de las Naciones Unidas (ONU) determinó metas e indicadores para, por un lado, evaluar el nivel de progreso y, por otro, hacer un seguimiento de la mejora de la calidad de vida de la población en los estados firmantes de este acuerdo. Y desde luego Marruecos fue uno de los países que prometieron alcanzar dichos objetivos, entre ellos dos dedicados a la mujer, lo que nos ha animado a abordar el tema de la igualdad de género y la salud materna en Marruecos. Así pues, trataremos la igualdad en nuestro país en materia de educación, empleo y adopción de decisiones, así como los cambios que ha experimentado el país a nivel normativo con relación a la mujer. Además, miraremos de cerca la salud materna en Marruecos debido a que esta se considera un verdadero problema en los países en vía de desarrollo.

\section{LA PROMOCIÓN DE LA IGUALDAD ENTRE GÉNEROS Y LA AUTONOMÍA DE LA MUJER}

La igualdad de género y los derechos de la mujer han sido un tema central a nivel internacional desde 1945, cuando se firmó la Carta de las Naciones Unidas en la Conferencia de las Naciones Unidas sobre Organización Internacional (Fernández y Melero, 2012). Marruecos, como miembro de las Naciones Unidas, ha participado en todas las etapas históricas en esta área. Dicho lo anterior, Marruecos ha avanzado notablemente en materia de 
género y de autonomía de las mujeres, resultado de varias reformas que ha vivido el país en las últimas décadas paralelas a un compromiso a nivel internacional, que requería una armonía en sus políticas y legislaciones respecto a las recomendaciones internacionales.

En este contexto, Marruecos participó en el advenimiento de la declaración de las Naciones Unidas para la erradicación de la violencia contra las mujeres, Plataforma de Acción de Beijing en 1995, e indudablemente se suscribió a la Declaración del Milenio y selló su compromiso para la consecución de los Objetivos de Desarrollo del Milenio (ODM), y ratificó la Convención sobre la Eliminación de Todas las Formas de Discriminación de la Mujer (Convention on the Elimination of all Forms of Discrimination Against Women, (CEDAW).

Por su lado, la nueva Constitución de 2011 supuso un avance y reformas en materia de igualdad. Así, esta constitución establece, en su artículo 19, que los hombres y las mujeres disfrutan de los mismos derechos y libertades civiles, políticas, económicas, culturales, sociales y medioambientales y, además, garantiza la obligación de velar por la igualdad entre ambos.

No cabe duda de que la situación de la mujer marroquí ha cambiado, lo que se refleja en los datos estadísticos, que confirman que las profundas reformas que ha conocido Marruecos durante las últimas décadas, tanto a nivel educativo, como laboral y político, han tenido repercusiones en la situación de ésta en especial y en la sociedad en general como veremos a continuación (Pérez, 2010).

\subsection{La igualdad en la educación}

Marruecos se comprometió, en materia de los ODM y la educación para todos, a eliminar la disparidad de género en todos los niveles de la enseñanza, así como a contribuir a la promoción de la igualdad de oportunidades para ambos sexos en general y en el sistema educativo en particular, y a generalizar la escolarización de las niñas tanto en el medio rural como en el urbano (HCP, 2017:42). En este contexto, el número de niñas que se matriculan hoy en día en las escuelas marroquíes con respecto a años anteriores confirma que se ha experimentado un cambio notable, pues en la actualidad muchas más niñas asisten a la escuela.

En efecto, examinando los datos que publica el Ministerio de Educación Nacional y de Formación Profesional cada año escolar denominados Recuel Statistique de l'éducation, extraemos que el índice de la paridad de género ha conocido una mejora notable en todos niveles de la enseñanza. Así, en cuanto a la enseñanza primaria en el sector público, la participación de las niñas pasó de un $86,51 \%$ en el año escolar 2002-2003 a un 90,09 \% en 2014- 2015, teniendo en cuenta que a principios de los años noventa ésta no superaba el $66 \%$. Por otra parte, esta mejora se ha registrado más en las zonas rurales, ya que la participación de las niñas aumentó en casi 8 puntos porcentuales en un período de once años, pasando de un $80,76 \%$ en el año escolar 2002-2003 a un 88,68\% en 2014-2015.

Por su lado, según los datos procedentes del Ministerio de Educación y de Formación Profesional, el índice de la paridad ha conocido una mejora continua, tanto en la enseñanza secundaria colegial, pasando de un 69,7\% en el año escolar 1990-1991 a un 75,2\% en 20012002 y a un $80,9 \%$ en 2013-2014, como en la enseñanza secundaria cualificada, que pasó de un 65\% en el año escolar 1990-1991 a un 90\% en 2013-2014 (HCP, 2015:42). A consecuencia de lo ocurrido en el ámbito de la enseñanza secundaria cualificada, el índice de la paridad de género en la educación superior pasó de un 56,6\% en 1990-1991 a un 93,3\% en 2013-2014, lo que significa que cada vez se está más cerca de lograr la igualdad en esta área $\mathrm{y}$, además, hay que señalar que en algunas especialidades como en el caso de los 
establecimientos de formación de ejecutivos, según el Haut Commissariat au Plan también referido como HCP (2015:43) este índice superó el 100\% (124,4 \% en 2013-2014) o en el ejemplo de la formación en el Instituto Nacional de Trabajo Social.

En definitiva, estas cifras de todos los niveles de la enseñanza demuestran evidentemente un progreso en el acceso de las mujeres a la educación. En este sentido, deseamos subrayar que este avance notable en la escolarización de las niñas en Marruecos se registró a partir de los años noventa, como indica Arjdal (2007), con la puesta en marcha de varios programas de desarrollo en las zonas rurales, tanto con la colaboración de las organizaciones internacionales, como con la de las asociaciones locales para superar el déficit en el ámbito social. Así, podemos mencionar el ejemplo del programa de apoyo a la escolarización de las niñas rurales, el de las prioridades sociales, el de abastecimiento agrupado de agua potable a las poblaciones rurales y la Iniciativa Nacional para el Desarrollo Humano, lo que permitió de manera directa o indirecta el aumento del número de niñas que acceden a las escuelas.

\subsection{La igualdad en el mundo laboral}

Empezaremos por indicar que antes de la independencia prácticamente la gran mayoría de las mujeres marroquíes eran amas de casa y las demás se dedicaban a lo que se consideraba una prolongación del trabajo doméstico y que consistía en trabajar en casas de colonos franceses o españoles, en fábricas u hospitales (Masson, 1938). No obstante, después de esta etapa hubo un cambio en la sociedad marroquí, de manera que, poco a poco, se posibilitó el acceso a otros tipos de trabajo, ampliándose así la participación de éstas.

Figura 1: la tasa de participación en la economía según sexo (\%)

\begin{tabular}{|c|c|c|c|}
\hline Años & Masculino & Femenino & Conjunto \\
\hline 1999 & 79,3 & 30,4 & 54,4 \\
\hline 2000 & 78,9 & 28,1 & 53,1 \\
\hline 2001 & 78,1 & 25,6 & 51,4 \\
\hline 2002 & 77,5 & 25,1 & 50,9 \\
\hline 2003 & 77,6 & 27,6 & 52,3 \\
\hline 2004 & 77,0 & 28,3 & 52,2 \\
\hline 2005 & 76,2 & 27,9 & 51,5 \\
\hline 2006 & 76,4 & 27,2 & 51,3 \\
\hline 2007 & 76,1 & 27,1 & 51,0 \\
\hline 2008 & 75,9 & 26,6 & 50,6 \\
\hline 2009 & 75,3 & 25,8 & 49,9 \\
\hline 2010 & 74,7 & 25,9 & 49,6 \\
\hline 2011 & 73,3 & 25,5 & 49,2 \\
\hline 2012 & 73,6 & 24,7 & 48,4 \\
\hline $\mathbf{2 0 1 3}$ & 73,0 & 25,1 & 48,3 \\
\hline
\end{tabular}

Fuente: Encuesta Nacional sobre el Empleo, HCP

Así pues, la situación de la mujer marroquí ha cambiado notablemente, pues actualmente está involucrada en casi todas las áreas, aunque es difícil hablar de una realidad unificada, ya que muchas realidades dependen de consideraciones como la clase social, el nivel cultural o el espacio geográfico (Chafai, 2007). Por otro lado, y en contraste con este cambio, hay que reconocer que desde el año 2000 la participación de las mujeres en las actividades 
económicas, como ha indicado el informe nacional de 2015 de los ODM, ha experimentado una tendencia descendente. De hecho, como se puede comprobar en la tabla (figura 1), la tasa de participación femenina en la economía marroquí pasó de un 30,4\% en 1999 a un 25,1\% en 2013, es decir, supuso una disminución en 5,3 puntos, en 2014 solo un 20,6\% de las mujeres trabajaban como asalariadas, teniendo en cuenta que esta tasa fue un 22,0\% en el año 2000.

Por otro lado, si bien es verdad que la tasa de desempleo femenino ha conocido una tendencia descendente, pasando del 13,2\% en 1999 al 9,6\% en 2013, cabe señalar que, desde otro punto de vista, en comparación con la tasa de desempleo masculino, que pasó de un $14,1 \%$ a un $9,1 \%$, la tasa femenina es elevada y además su ritmo de disminución es menor. En otras palabras, en desempleo masculino disminuyó en 5 puntos porcentuales mientras que el femenino solo en 3,6 puntos.

Por otra parte, como se puede comprobar en la tabla (figura 2), el análisis de la tasa de desempleo según el nivel de estudios y género refleja que la tasa de desempleo femenino es más alta en comparación con la del masculino, tanto respecto a la población con un nivel de estudios medio, como a la que tiene uno superior. Sin embargo, en cuanto al desempleo de la población sin ningún diploma, se registra que, por un lado, la tasa de desempleo femenino es muy baja $(2,6 \%$ en 2013) y, por otro, es la única categoría donde no supera a la tasa desempleo masculino. Por un lado, esto demuestra que las mujeres con estudios tienen más dificultad para encontrar un puesto de trabajo que los hombres, al contrario de las que no tienen estudios. Esta situación confirma que la mayoría de las mujeres se dedican a actividades que no requieren estudios o cualificaciones.

Figura 2: La tasa de desempleo según sexo y nivel de estudios (\%)

\begin{tabular}{|c|c|c|c|c|c|c|c|c|}
\hline Año & \multicolumn{3}{c}{ Sin diploma } & \multicolumn{2}{c}{$\begin{array}{c}\text { Nivel } \\
\text { medio }\end{array}$} \\
\cline { 2 - 10 } & F & M & F & M & F & M & F & M \\
\hline 1999 & 5,5 & 9,1 & 34,7 & 24,9 & 36,2 & 23,5 & 13,2 & 14,1 \\
\hline 2000 & 4,3 & 8,1 & 33,0 & 25,3 & 39,2 & 23,9 & 12,8 & 13,6 \\
\hline 2001 & 4,1 & 7,1 & 30,1 & 22,8 & 35,5 & 22,6 & 12,2 & 12,3 \\
\hline 2002 & 3,8 & 6,0 & 29,2 & 20,7 & 35,3 & 22,1 & 12,1 & 11,1 \\
\hline 2003 & 4,0 & 6,1 & 30,5 & 20,3 & 36,8 & 22,3 & 12,4 & 11,2 \\
\hline 2004 & 3,3 & 5,8 & 29,0 & 19,4 & 35,1 & 22,3 & 11,1 & 10,7 \\
\hline 2005 & 3,1 & 6,1 & 29,4 & 19,1 & 35,6 & 22,0 & 11,3 & 11,0 \\
\hline 2006 & 3,1 & 5,6 & 25,2 & 17,3 & 27,1 & 15,7 & 9,7 & 9,7 \\
\hline 2007 & 3,1 & 5,6 & 23,6 & 16,9 & 28,5 & 16,8 & 9,8 & 9,8 \\
\hline 2008 & 2,9 & 5,5 & 23,6 & 16,8 & 28,2 & 14,9 & 9,8 & 9,5 \\
\hline 2009 & 2,8 & 5,1 & 22,8 & 15,5 & 26,0 & 14,2 & 9,5 & 9,0 \\
\hline 2010 & 3,2 & 5,0 & 22,1 & 14,8 & 25,3 & 14,3 & 9,6 & 8,9 \\
\hline 2011 & 2,8 & 4,5 & 22,8 & 14,0 & 28,5 & 14,4 & 10,2 & 8,4 \\
\hline 2012 & 2,8 & 4,6 & 21,0 & 14,3 & 27,4 & 14,0 & 9,9 & 8,7 \\
\hline 2013 & 2,6 & 5,2 & 20,0 & 14,2 & 26,7 & 14,8 & 9,6 & 9,1 \\
\hline
\end{tabular}

Fuente: elaboración propia a partir de los datos de encuesta nacional sobre empleo, HCP. 
En efecto, no nos extraña que las mujeres estén más presentes en el sector de la agricultura, la silvicultura y la pesca con una cuota del 59,9\% del empleo femenino a nivel nacional, sobre todo en el medio rural $(93,2 \%)$. Por otro lado, debemos señalar que estas actividades en el mundo rural a menudo carecen de remuneración, baste mencionar que un $73,6 \%$ se dedican a estos trabajos impagados. Pero esto no quiere decir que se trate de una cuestión rural, sino de una situación global, tanto en el medio rural como en el urbano, ya que las condiciones sociales y laborales son precarias, se caracterizan por la inestabilidad dado que asiduamente son trabajos temporales o parciales con bajos sueldos. Además, frecuentemente no se benefician de ninguna cobertura médica, baste indicar que un $81,6 \%$ de las mujeres ocupadas no tienen este servicio (HCP, 2013:11).

Finalmente, considerando esta situación de la mujer y para aumentar su participación en las actividades económicas, varios departamentos y entidades ya han puesto en marcha algunas acciones y programas, entre ellos la Iniciativa Nacional para el Desarrollo Humano que se focaliza en las mujeres en situación de gran precariedad y sin recursos.

\subsection{La participación en los procesos de toma de decisiones}

En primer lugar, cabe resaltar que la situación social de la mujer marroquí ha conocido un salto notable, sobre todo desde un punto de vista cualitativo, aunque este cambio puede parecer pausado desde la mirada occidental (Vallejo, 2010). Por una parte, debido a una cultura tradicional dominante en una sociedad patriarcal y, por otro, a los avances limitados en el ámbito de la educación de las mujeres, como indica Enhaili (2006), que a nuestro entender explicaría el porcentaje débil de las mujeres que consideraban que la política es "muy importante" o "importante" en la vida (Hilal y Vallejo, 2016), la participación de la mujer marroquí en las élites políticas era débil, tanto a nivel local como nacional, y por lo tanto, no formaban parte del proceso de adopción de decisiones a pesar de su involucración en diferentes luchas políticas y sociales.

Marruecos se ha comprometido a que las mujeres gocen de sus derechos plenamente a través de la adopción de reformas jurídicas y de estrategias de desarrollo con el fin de atenuar o eliminar las desviaciones que perpetúan las discriminaciones basadas en el género. En este contexto, la nueva Constitución de 2011 establece en su artículo 154 que "los servicios políticos se organizan sobre la base del acceso igual de las ciudadanas y de los ciudadanos", lo que quiere decir que el principio de igualdad y equidad está institucionalizado en el disfrute de los derechos políticos.

Téngase en cuenta que a pesar de que la mujer marroquí recibió el derecho a votar y a ser candidata en 1963, el país era objeto de críticas por la exclusión de las mujeres de las instituciones representativas (Pérez, 2008), teniendo que esperar la primera parlamentaria hasta 1993. En la actualidad, y para mejorar su representación en el panorama político marroquí, la Ley Orgánica n ${ }^{\circ} 27-11$ de 14 de octubre de 2011 relativa al acceso a la Cámara de Representantes, establece la aplicación del principio de discriminación positiva, ya que le dio una cuota de 60 asientos para las mujeres de un total de 395, esto es, un 15\%. Así, el número de mujeres electas en las elecciones de noviembre de 2011 llegó a 67 parlamentarias, implicando que las mujeres ocupan casi un $17 \%$, un 16,96\%, de los escaños en el parlamento marroquí, pero deberíamos mencionar que se trata de una tasa inferior a la media a escala mundial situada en un 20,3\% (Sahuquillo, 2013).

Por otro lado, en las elecciones municipales y regionales de septiembre de 2015, la tasa de las mujeres llegó a un 21,64\% respecto a los candidatos de las elecciones municipales, y a un 
$38,64 \%$ en cuanto a las elecciones regionales. Estas mujeres ganaron 6.673 de los escaños a nivel de los municipios, lo que supone una tasa de un $21,17 \%$.

Estos datos creemos que es verdad que muestran una mejora cuantitativa si los comparamos con los resultados de las anteriores elecciones, ya que por ejemplo el número de escaños ganados por las mujeres se dobló entre las elecciones de 2009 y las de 2015. Sin embargo, las cifras demuestran que, a pesar de las reformas, la representación de la mujer marroquí todavía es insuficiente si tenemos en cuenta que la población femenina marroquí representa un $56,8 \%$ de la población total, y sólo constituye un $45 \%$ de los marroquíes inscritos en las listas electorales.

\subsection{Modificaciones a nivel del arsenal jurídico}

No cabe duda de que la igualdad de géneros en cualquier sociedad supone un reconocimiento de los derechos de todos los ciudadanos, sin ninguna discriminación que les impida compartir el mismo protagonismo en esta. Esto, por su parte, no sólo se considera un derecho humano principal, sino que va más allá, ya que puede significar el fundamento de una sociedad próspera, pacífica y sostenible. En este contexto, en la sociedad marroquí, la mujer cada vez está jugando un rol social y político más influyente (Pérez, 2008). No obstante, cabe señalar que esto requirió unos cambios y modificaciones a muchos niveles, entre ellos a nivel normativo, que es un factor de gran importancia en el ámbito de la igualdad entre hombres y mujeres.

En efecto, se han modificado varias leyes como el Código de Familia (Mudawana), el Código Penal, el Código de Trabajo, el Código de la Nacionalidad, el Código Electoral y la Carta Comunal. Además de estas modificaciones, la nueva Constitución de 2011, establece que la mujer, al igual que el hombre, goza de todos los derechos y libertades de carácter civil, político, económico, social y medioambiental. Asimismo, en el preámbulo de esta Constitución se insiste en la interdicción y la lucha contra todas formas de discriminación por razón de género, raza, confesión, cultura, pertenencia social o regional, idioma, discapacidad o cualquiera otra situación personal.

Así, en el terreno de la igualdad, el artículo 30 de la Constitución establece la necesidad de prever en la ley "las disposiciones que por su naturaleza favorecen el acceso igual de las mujeres y de los hombres a las funciones electivas", de la misma manera que el artículo 146 relativo a las regiones y a las colectividades territoriales aborda el mismo tema estableciendo que una ley deberá fijar "las disposiciones destinadas a garantizar una mayor participación de las mujeres en los consejos territoriales". Por lo tanto, están establecidos o se van a establecer algunos mecanismos u organismos.

Para contribuir a la difusión de los principios de equidad, de igualdad y de mejora de la imagen de la mujer marroquí, el 15 de marzo de 2005 se adoptó la Carta Nacional para la mejora de la imagen de la mujer en los medios de comunicación marroquíes, debido a que se presentaba una imagen estereotipada de la mujer en la mayoría de estos medios, limitada a unos modelos específicos basados en la mujer tradicional o mujer objeto ignorando su rol real en la sociedad marroquí. Por ello, esta Carta hizo un llamamiento a todos estos medios para que aplicasen el enfoque de género. Asimismo, en 2015 se creó un Observatorio con el mismo nombre de la carta para velar por el cumplimiento de lo estipulado.

Por otro lado, como establece el artículo 19 y el 164 de la Constitución de 2011, es necesaria la creación de la Autoridad para la paridad y la lucha contra todas las formas de discriminación. En consecuencia, el parlamento marroquí aprobó el proyecto de Ley 79.14, relativa a la Autoridad para la paridad y la lucha contra todas las formas de discriminación, como el órgano encargado del seguimiento de la evaluación de las políticas públicas relativas 
a la promoción de los derechos de la mujer y la lucha contra todas las formas de discriminación. A pesar de esto, cabe señalar que las feministas marroquíes lo rechazaron debido a sus "competencias limitadas", mientras que la ministra de Solidaridad, Mujer, Familia y Desarrollo Social señaló que "este paso se consideraba una victoria de la lucha feminista, de la mujer marroquí, de la constitución marroquí y de Marruecos en general".

Para la lucha contra todas las formas de discriminación y de violencia contra las mujeres, la Ley 14-05 relativa a las condiciones de apertura y gestión de las instituciones de protección social, aprobada en 2006, se revisó tras la publicación del informe del Ministerio en 2014 para incluir las residencias femeninas de estudiantes dar taliba e instituciones de acogida de mujeres y niñas víctimas de violencia. Esta revisión, como indicó la ministra de Solidaridad, Mujer, Familia y Desarrollo Social, Bassima Hakkaoui, "es el primer paso en el proceso de reforma de estas instituciones, cuyo fin es lograr la eficiencia, eficacia y gobernanza".

\section{LA MEJORA DE LA SALUD MATERNA}

\subsection{El serio problema de la mortalidad materna en países en vías de desarrollo}

Como punto de partida deberíamos recordar que la Organización Mundial de Salud (OMS) define la mortalidad materna como "la muerte de una mujer mientras está embarazada o dentro de los 42 días siguientes a la terminación del embarazo, independiente de su duración y lugar, debida a cualquier causa relacionada con la gestación -o agravada por ésta- o con su forma de atención, pero no por causas accidentales o incidentales". Esta mortalidad ha sido y sigue siendo un verdadero problema, debido a que es el primer motivo de fallecimiento en mujeres en edad reproductiva a nivel mundial. Además, es un indicador importante de desarrollo humano y social que refleja, en gran medida, la situación de la mujer y sobre todo de su acceso a la sanidad en cualquier país. Por ello, se determinó como quinto objetivo de los ODM la mejora de la salud materna, con la meta de reducir en un $75 \%$ entre 1990 y 2015 la mortalidad de las mujeres por esta causa.

A pesar de que en muchos casos las cifras de la mortalidad materna quedan lejos de la realidad, ya que medir esta mortalidad no es una tarea fácil sobre todo cuando se trata de los países en desarrollo donde los datos que se precisan no se registran de forma sistemática (UNICEF, 2008:5), es evidente que hay una mejora en la evolución de la tasa de la mortalidad materna. En efecto, su tasa pasó de 385 por cada 100.000 nacidos vivos en 1990 a 221 en 2014, según los datos del Banco Mundial. No obstante, deberíamos tener en cuenta que en los países en vía de desarrollo todavía se considera un verdadero problema que urge solucionar, baste indicar que el $99 \%$ de la mortalidad materna a nivel mundial ocurre en estos países (OMS, 2015).

\subsection{La evolución gradual en la mejora de salud materna en Marruecos}

La iniciativa para la maternidad sin riesgo es un esfuerzo mundial para combatir la mortalidad y morbilidad materna. Su objetivo es mejorar la calidad de vida de las mujeres y la eliminación de los riesgos a través de la adopción de un conjunto de estrategias sanitarias y no sanitarias. Esta iniciativa hizo hincapié en la necesidad de asegurar un acceso más amplio a los servicios de salud materna de calidad, de desarrollar los servicios de planificación familiar, así como de adoptar medidas eficaces para mejorar la condición y el estatuto de las mujeres. 
En el caso de Marruecos, los principales programas desarrollados por el Ministerio de Salud en los años setenta se dirigieron principalmente a la salud de los niños como objetivo prioritario para la reducción de la mortalidad infantil. Así pues, en 1970 se realizó un programa de Protección Materna e Infantil (Protection Maternelle et Infantile, PMI), en el que se da prioridad a los niños, y se estableció en un decreto ministerial la atención prenatal en todas las unidades sanitarias ambulatorias. En 1974 se cambió el PMI por el programa de Protección de la Salud del Niño (Protection de la Santé de l'Enfant, PSE) y, además, se hizo la codificación de las consultas prenatales, a saber, tres visitas prenatales una postnatal. En este contexto, cabe señalar que en aquella época Marruecos conocía una gran escasez de personal sanitario o cualificado, sobre todo para la atención a mujeres embarazadas, como era el caso de las parteras, cuyo número en todo el país no superaba las doscientas. Tres años más tarde, en 1977 se estableció un programa de Protección de la Salud de la Madre y del Niño (Protection de la Santé de la Mère et de l'Enfant, PSME). Si comparamos el nombre de este programa con el del anterior, podemos observar una evolución en el concepto al incluir a la progenitora considerándose así la salud de ambos, madre e hijo, inseparable ya que, como bien señala UNICEF "un niño sano requiere una madre sana".

A partir de 1987 hubo un avance en esta área, pues se desarrolló una Estrategia Nacional entre 1988 y 1995 con el objetivo general de obtener unas tasas satisfactorias respecto a la cobertura prenatal y de partos con el fin de reducir la mortalidad materna y la perinatal. Este avance empezó con el establecimiento del Programa de Vigilancia del Embarazo y del Parto (Programme de Surveillance de la Grossesse et de l'Accouchement) que se centró en la reducción de la mortalidad y morbilidad materna y, por primera vez, se determinaron objetivos cuantitativos de cobertura prenatal y de parto vigilado. Además, con este programa se introdujo la vacuna antitetánica, lo que supuso, por su lado, la valorización de las consultas prenatales, y la sensibilización de parteras, posterior a la realización de un censo de éstas, beneficiando así a unas 13.000 parteras tradicionales.

Asimismo, se lanzó un proyecto de Fondo de Población de las Naciones Unidas para el apoyo de las actividades de vigilancia del embarazo y del parto, para contribuir al fortalecimiento y la integración de las actividades de la Maternidad Sin Riesgo y de la Planificación Familiar en todo el país, sobre todo en las provincias de Azilal, Errachidia, Tata y Ouarzazat, pues presentan obstáculos, entre ellos geográficos, que les dificultan la accesibilidad y cuentan con indicadores sanitarios desfavorables. Por otra parte, la creación en 1990 de un servicio específico a la protección de la salud materna demostró la voluntad para la reducción de la mortalidad materna en Marruecos.

A partir de 1995, hubo un cambio estratégico decisivo en la política del Ministerio de Salud en el tema de la reducción de mortalidad materna. En efecto, la prioridad esta vez se dio al mejoramiento de las maternidades hospitalarias y de las rurales y a las casas de parto (des maisons d'accouchements).

Sin embargo, todo lo que hemos mencionado no nos impediría afirmar que las cifras relacionadas con la mortalidad materna en aquella época reflejaban una realidad dolorosa y muy preocupante, ya que la tasa de mortalidad materna a nivel nacional para el período 19851991 se estimaba en 332 por cada 100.000 nacidos vivos.

Según la Encuesta Nacional sobre la Salud de la Madre y del Niño de 1997 (Enquête Nationale sur la Santé de la Mère et de l'Enfant, ENSME, 1997) esta tasa llegaba a 228 por cada 100.000 nacidos vivos para el período 1992-1997. Además, esta tasa experimentó una disparidad notable entre las zonas rurales y urbanas, pues la tasa de mortalidad materna entre las mujeres rurales llegaba a 307 por cada 100.000 nacidos vivos, mientras que entre las urbanas llegaba a 125, reflejando así la gran diferencia en cuanto a la condición de vida de 
las mujeres en ambas zonas. Afortunadamente, la evolución de estas tasas en Marruecos es favorable, esto es, se va reduciendo, ya que según la Encuesta Nacional Demográfica de 2009-2010 (l'Enquête Nationale Démográphique à Passages Rèpétes, ENDPR), esta tasa bajó a 112 muertes maternas por cada 100.000 nacidos vivos, 73 entre las mujeres urbanas y 148 entre las rurales, o lo que es lo mismo, en este período entre las dos encuestas la tasa de mortalidad maternal rural ha disminuido en un $51,7 \%$ y la urbana en un $41,6 \%$. Esto se explicaría por los esfuerzos realizados sobre todo después de fijar los ODM, ya que después del año 2000 se han adoptado varias medidas. Entre ellas, conviene subrayar que, al Programa Nacional de Planificación Familiar lanzado en 1966, que logró resultados inmediatos sobre todo a partir de los años ochenta tanto a nivel demográfico como a nivel sanitario (El-Arbi, 2005), a partir de 2001 se le otorgó autonomía total en materia de compra de insumos anticonceptivos y de adquisición de equipos médicos y técnicos a partir de 2005 . A causa de esta media, la tasa de mujeres casadas (entre 15 y 49 años) que usan anticonceptivos ha conocido un aumento notable, dado que esta tasa, según el Ministerio de Salud, pasó de un 20\% (una mujer de cada cinco) en 1979-1980 a un 62,6\% (más de 3 mujeres de cada 5) en 2003-2004, y a un 67,4\% en 2011.

Por su parte, en 2006 se elaboró la Libreta de Salud de la Mujer (Carnet de Santé de la Femme), que es un soporte de información donde se debe registrar todos los datos relativos a salud de la madre, al control de su embarazo, al parto y sus seguimientos. La elaboración de esta libreta se considera, desde nuestro punto de vista, un paso positivo en materia de salud materna, ya que así se puede recoger un resumen de datos relevantes de la vida sanitaria de la mujer y, a su vez, puede ser una herramienta de comunicación entre médicos, enfermeros y parteras que intervienen en la atención preventiva y curativa de ésta. No obstante, sería más útil, a nuestro entender, si esta libreta tuviese un formato digital, pues de este modo estaría siempre a disposición de los profesionales, sería más fácil de consultar y disminuiría la posibilidad de su extravío. Por otro lado, debemos cuestionar si realmente este documento está generalizado, es decir si todas las mujeres de entre 15 y 49 años están en posesión de éste y hacen uso de él. Desgraciadamente, a pesar de la carencia de datos oficiales del Ministerio de Salud, podemos subrayar que no todas las mujeres lo poseen. Prueba de ello es que un $37,3 \%$ de las mujeres embarazadas en mundo rural marroquí no reciben ninguna atención prenatal, como se puede concluir de los datos de los indicadores de la realización de los programas de la Salud Maternal, Infantil y de la Planificación Familiar.

Por otro lado, uno de los mayores problemas que afectan a la salud de las mujeres a nivel mundial es el cáncer de mama y el de cuello de útero, ya que son enfermedades muy frecuentes entre éstas. Marruecos no es ninguna excepción en este respecto, de hecho, según el registro de cánceres de 2004, en la región de Gran Casablanca estos dos tipos mencionados representan la mitad de los cánceres femeninos (Ministère de la Santé et Association Lalla Salma contre le Cancer, 2011:10). Además, como podemos comprobar en el gráfico (figura 3 ), el cáncer de mama representa la primera causa de mortalidad por cáncer entre mujeres (alrededor de un 28\%) seguido por el cáncer de cuello de útero (alrededor de un 10\%).

En este sentido, el rasgo más singular que caracterizaba la política sanitaria frente a estas enfermedades femeninas era la ausencia de programas de detección sistemática antes de 2010. Por ello, las repercusiones de esta carencia fueron muy negativas, por ejemplo, dos de cada tres de casos de cáncer de cuello del útero se diagnosticaban en fases muy avanzadas. Sin embargo, a partir de esta fecha se empezó la integración progresiva de la detección precoz de cáncer de mama y de cuello de útero en las actividades de salud reproductiva. Así, se lanzó un programa en este ámbito con el fin de mejorar la atención de las mujeres que padecen estos dos cánceres a través de un diagnóstico precoz y tratamiento. 
Figura 3: Distribución de la mortalidad por cáncer entre las mujeres, Marruecos

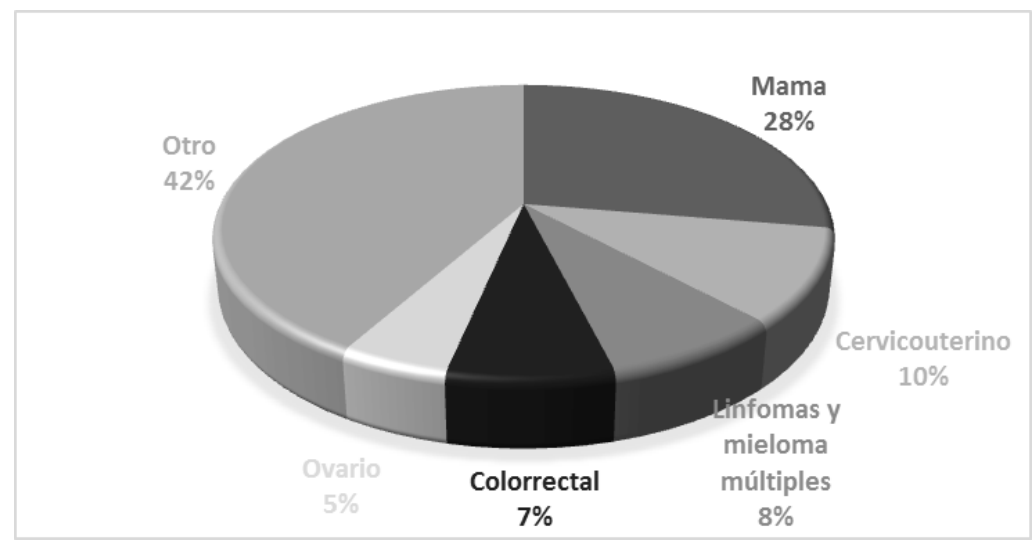

Fuente: Organización Mundial de la Salud. Perfiles oncológicos de los países, 2014

Como resultado de estos esfuerzos mencionados, ha habido una evolución favorable en cuanto a algunos indicadores relacionados con la salud materna, lo que, sin duda, ha contribuido a la disminución de la mortalidad materna en Marruecos, como se puede inferir de la siguiente tabla (figura 4).

Figura 4: Factores contribuyentes en la disminución de la mortalidad materna

\begin{tabular}{|l|c|c|c|c|c|c|}
\cline { 2 - 7 } \multicolumn{1}{c|}{} & \multicolumn{2}{c|}{ Nacional } & \multicolumn{2}{c|}{ Urbano } & \multicolumn{2}{c|}{ Rural } \\
\hline Factores & $1999-2003$ & $2009-2010$ & $1999-2003$ & $2009-2010$ & $1999-2003$ & $2009-2010$ \\
\hline $\begin{array}{l}\text { Tasa total de } \\
\text { fecundidad }\end{array}$ & 2,7 & 2,2 & 2,2 & 1,8 & 3,4 & 2,7 \\
\hline $\begin{array}{l}\text { Cobertura de } \\
\text { atención } \\
\text { prenatal (\%) }\end{array}$ & 67,8 & 80,2 & 84,9 & 94 & 47,9 & 68,3 \\
\hline $\begin{array}{l}\text { Asistencia } \\
\text { en parto }\end{array}$ & 62,6 & 74,1 & 85,3 & 93 & 39,5 & 56,7 \\
\hline
\end{tabular}

Fuente: HCP, 2011

En efecto, entre los períodos 1999-2003 y 2009-2010, la asistencia en el parto a nivel nacional aumentó en unos 11,5 puntos porcentuales, pasando de un $62,6 \%$ a un $74,1 \%$. Sin embargo, llama atención la baja tasa en el mundo rural que, a pesar de su aumento en unos 17,2 puntos porcentuales, todavía supone una tasa modesta y refleja que en el mundo rural marroquí aun casi de la mitad de los partos ocurren sin ninguna asistencia, constituyendo un peligro para la salud y vida de la mujer y del niño. Esto indudablemente no siempre está causado por la dificultad de acceso a las instalaciones sanitarias, sino que a veces se trata de una falta de sensibilización. En otras palabras, si no es posible satisfacer la necesidad de toda la población rural en materia de salud materna, al menos esta población debería tener consciencia de la repercusión negativa del parto en hogares particulares. 
De igual modo, la cobertura de atención prenatal ha aumentado en 12,4 puntos porcentuales a nivel nacional, pasando en el medio urbano de un $84,9 \%$ a $94 \%$. Por su lado, la tasa de fecundidad ha conocido una evolución favorable, ya que esta tasa ha pasado de 2,7 a 2,2 a nivel nacional, acentuándose más esta disminución en el mundo rural pasando de 3,4 a 2,7 .

Así, en 2015, fecha que se acordó para lograr los ODM, según un informe conjunto de la OMS, el UNICEF, el UNFPA, el Grupo del Banco Mundial y la División de la Población de la ONU publicado a finales de 2015, la tasa de mortalidad materna ha llegado a 121 por cada 100.000 nacidos, lo que significa una tasa de disminución de un 61,8\% entre 1990 y 2015 , quedando cerca de la media mundial (216) y muy por debajo de la media del continente africano, que conoce una tasa de mortalidad materna muy elevada (495). Además, si comparamos esta tasa con la de otros países árabes, observamos que el gráfico (figura 5) refleja que Marruecos se considera uno de los países con una tasa media a nivel de los anteriormente mencionados, representa una tasa muy baja si la comparamos con países como Mauritania, Yemen, Sudan, o Yibuti, y muy elevada si la comparamos con la de Catar, Bahrein y Egipto. Asimismo, se observa que en el período fijado para lograr los ODM, el ritmo de disminución de esta tasa en Marruecos ha sido más acelerado en comparación con sus países vecinos, ya que en nuestro país ha disminuido en un $61,8 \%$, en Argelia en un $35,8 \%$ y en Mauritania en un 29,9\%.

Esto explica la mención en dicho informe de que Marruecos es un país making progress (haciendo progresos). No obstante, si volvemos a las metas determinadas para el objetivo 5 de los ODM, encontramos que se marcó la reducción de la mortalidad materna con el fin de llegar a sólo 83 casos por cada 100.000 nacidos vivos.

Figura 5: Tasa de mortalidad materna en algunos países árabes (por cada 100.000 nacidos vivos)

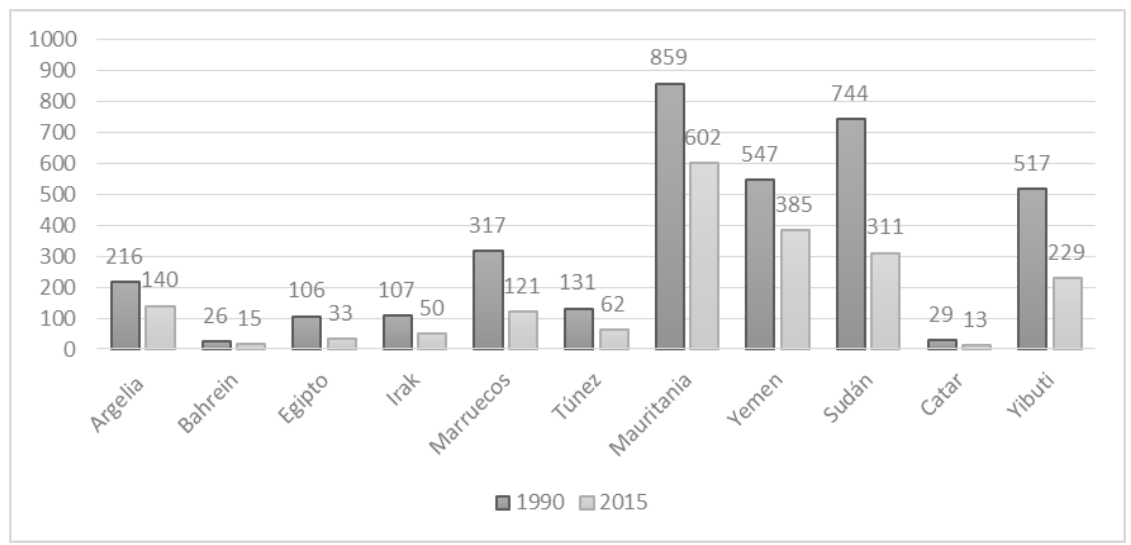

Fuente: Elaboración propia a partir de los datos de la OMS, el UNICEF, el UNFPA, el Grupo del Banco Mundial y la División de la Población de la ONU en conjunto

Basándonos en los datos del Banco Mundial, podemos decir que es verdad que ha habido una evolución positiva, pero no se ha logrado este propósito, prueba de esto es que esta tasa llegó en 2015 a 121 casos por cada 100.000 nacidos vivos. 


\section{CONCLUSIÓN}

Es evidente que lograr un desarrollo global depende de varios factores y, en este sentido, las experiencias han demostrado que la mujer forma un pilar relevante para su consecución. Así, desde de los años setenta encontramos algunas estrategias que promueven el rol de la mujer, sirvan de ilustración Mujer En Desarrollo (MED) y Género En Desarrollo (GED). Y con el comienzo de este siglo, la Comunidad Internacional estableció los OMD, que podemos considerarlos como un mecanismo de control y medición de los avances realizados por cualquier país, entre ellos los avances en igualdad de género y salud materna, que según hemos citado anteriormente constituyen dos objetivos de los OMD que hemos intentado abarcar en este artículo.

Como hemos visto, Marruecos ha conocido varias reformas que han contribuido a lograr unos avances en materia de igualdad de género y de autonomía de las mujeres. No obstante, debemos registrar que todavía existen varios escollos que se debe soslayar. En este contexto, apuntamos que no es suficiente establecer leyes o llevar a cabo reformas, sino que lo más importante su posterior aplicación. A pesar de que la constitución marroquí menciona en 6 ocasiones la palabra igualdad, sin embargo, se observa una lentitud en la aplicación de las exigencias de este texto. Por otra parte, creemos que las colectividades territoriales deben desempeñar un papel de más peso realizando mayores esfuerzos, pues, de hecho, no encontramos el tema de la igualdad de género en los ejes estratégicos del Plan Comunal de Desarrollo de algunas comunas. Asimismo, si bien es verdad que la situación laboral de la mujer marroquí ha conocido un cambio positivo tanto cuantitativo como cualitativo, no obstante, las mujeres todavía se dedican a trabajos no remunerados ni gozan de cobertura social sobre todo en el mundo rural.

En cuanto a la disminución de la mortalidad materna, que ha conocido una disminución sin llegar a los niveles marcados como uno de los objetivos de ODM como hemos observado, esto se explica, a nuestro entender, por las dificultades que todavía sufre la mujer, especialmente en mundo rural, para acceder a los servicios básicos, como el caso de los servicios sanitarios. Esto repercute negativamente en la aceleración de la disminución de la tasa de mortalidad materna, baste indicar que ésta en el mundo rural marroquí duplica el número de la de las zonas urbanas. El hecho de que el mundo rural padezca más este problema, no es sorprendente si tomamos en consideración la carencia de infraestructuras de base en zonas rurales, ya que, a veces, la ubicación de los centros hospitalarios y el mal estado de caminos son factores determinantes fatales. Por ello, creemos que la solución necesaria pasa por la mejora de las infraestructuras de base en el mundo rural y la sensibilización de la importancia del control sanitario de los alumbramientos, ya que más de la mitad de los partos en el mundo rural marroquí ocurren sin ningún control médico. Además, se debe proporcionar tanto recursos humanos cualificados como materiales suficientes para poder dar un servicio que se adecue a las necesidades de las pacientes.

\section{BIBLIOGRAFÍA}

Arjdal, M. (2007) "La escolarización de la niña rural y su rol en el desarrollo humano". Dialogo Civilizado, no 1863, 23-03-2007 (en árabe), en: https://goo.gl/5az7SU (consulta 03/02/2017).

Chafai, L. (2007) "Las mujeres sujeto de marginación en Marruecos". Anales de Historia Contemporánea, $\mathrm{n}^{\circ} 13,1997$. pp. 35-36 en: https://goo.gl/LrjeM4 (consulta 06/02/2017). 
El-Arbi, H. (2005) "Planification familiale". En Ministère de la Santé. Enquête sur la Population et la Santé Familiale (EPSF), Maroc. 2003-2004. Mars. 2005, pp.57-81.

Enhaili, A. (2006) "Femmes, développement humain et participation politique au Maroc". En: https://goo.gl/oEWLxm (consulta 08/02/2017).

Fernández Alles, J. y Melero Aguilar, N. (2012) "Igualdad de género y desarrollo humano en Marruecos: Cuestiones pendientes tras la Constitución de 2011”. Revista de Estudios Internacionales Mediterráneos, $\mathrm{n}^{\circ} 13$ (julio-diciembre 2012), en: https://goo.gl/sxjfhD (consulta 02/02/2017).

Haut Commissariat au Plan, HCP (2013) Femmes Marocaines et Marché du Travail: Caractéristiques et Évolution. Décembre 2013.

Haut Commissariat au Plan, HCP (2015) Le Maroc entre Objectifs du Millénaire pour le Développement et Objectifs de Développement Durable: Les acquis et les défis. Rapport National 2015, Août 2015.

Hilal, L. y Vallejo Peña, F. A. (2016) "Mujer marroquí y modernización social". Barataria: Revista Castellano-Manchega de Ciencias Sociales, 21:39-57, DOI: http://dx.doi.org/10.20932/barataria.v0i21

Ministère de las Santé et Association Lalla Salma contre le Cancer (2011) Guide de Détection Précoce des Cancers du Sein et du Col de l'Utérus.

Organización Mundial de la Salud, OMS (2015) Naciones Unidas: la mortalidad materna se ha reducido un 44\% desde 1990, en: https://goo.gl/i7YvKE (consulta/ 03/03/2017).

OMS (2013) Nota de Prensa. Enfermedades diarreicas. Nota descriptiva $\mathrm{N}^{\circ} 3330$. Abril de 2013, en: https://goo.gl/TQbNu7 (consulta 12/02/2017).

Pérez Beltrán, C. (2008) "Mujeres marroquíes en la vida pública: entre el cambio social y la identidad musulmana". En: M. D. López Enamorado (ed.) España y Marruecos: Mujeres en el espacio público. Sevilla: Alfar, pp. 91-109.

Pérez Beltrán, C. (2010) "Las mujeres en el medio rural marroquí: un estudio de caso sobre la alfabetización de mujeres en el círculo rural de Asila". Anaquel de Estudios Árabes, 21: 55-75.

Sahuquillo, M. R. (2013) "El número de parlamentarias aumenta por la aplicación de cuotas". El País, en: https://goo.gl/6ts1Yg (consulta 10/02/2017).

UNICEF. (2008) Estado Mundial de la Infancia 2009: Salud Materna y Neonatal. Diciembre de 2008.

Vallejo Peña, F. (2010) "Evolución reciente de la participación política y social en la mujer marroquí: un análisis a través de la encuesta mundial de valores (2001-2007)". Revista Internacional de Pensamiento Político, I Época, 5: 287-306.

\section{Breve currículo:}

\section{Mohamed Laghdas}

Doctorando en el Departamento de Geografía Humana de la Universidad de Granada (España), sobre la experiencia de Marruecos en Desarrollo: el caso de la INDH. DEA en Derecho Ambiental en Andalucía: nuevos sectores de Turismo, Ocio y Deporte (UGR) y Diploma de Especialista Universitario en la Gestión del Patrimonio (Universidad de Alicante, España). Licenciado en Desarrollo Local y Ordenación Territorial (Universidad Abdelmalek Essaadi de Tetuán, Marruecos) y Licenciado en Geografía (Universidad Abdelmalek Essaadi de Tetuán, Marruecos), con formación adicional en el ámbito del desarrollo y del patrimonio (Curso F.P.O. Agente Dinamizador del Medio Rural, Formación en Patrimonio histórico), participante y ponente en conferencias y congresos, así como colaborador en la realización de proyectos de desarrollo e intervención social en España y en el norte de Marruecos (Proyecto Infancia en Dificultad Social -Menores Inmigrantes No Acompañados en Cruz Roja Española Granada y Agente de desarrollo en el diagnóstico participativo Territorial en la provincia Fahs Anjra, Tánger). 